Jpn. J. Health Phys., 54 (1), $72 \sim 73$ (2019)

\section{From Japan to the World}

\section{J-EPISODE: Japanese Epidemiological Study on Low-Dose Radiation Effects Conducted by the Radiation Effects Association}

\author{
Institute of Radiation Epidemiology, \\ Radiation Effects Association*1
}

\section{Background}

The Radiation Effects Association (REA), founded in 1960, conducts, promotes, and subsidizes research to investigate the biological and environmental effects of low-dose radiation and also informs the general public of these effects. The REA now covers two areas of investigation: registering and managing nuclear workers' exposure doses and conducting epidemiological radiation studies on nuclear workers.

To conduct an epidemiological survey of Japanese nuclear workers, the REA established the Institute of Radiation Epidemiology in 1990 and has been performing epidemiological investigations since then. The decision to establish the institute was encouraged by the Science and Technology Agency (later the Ministry of Education, Culture, Sports, Science and Technology) and the movement of the International Agency for Research on Cancer at that time.

It is well known that the epidemiological study of atomic bomb survivors that has been ongoing since 1950 is the gold standard for studies on radiation effects. However, this study includes the health effects of single, acute exposure to relatively high-dose and high-dose-rate radiation; an extrapolation to low-dose and low-dose-rate radiation is required to apply the practical doses of radiation protection (that is, an order over ten $\mathrm{mSv}$ ) in normal operation. To carry out a new epidemiological survey study to examine nuclear workers in Japan, it was necessary to design an original framework for the survey and to establish a protocol to allow for long-term survey administration.

\section{Protocol}

\subsection{Establishment of study cohort}

The study cohort included mainly nuclear power plant workers with Japanese nationality who were engaged in radiation work during normal operation at nuclear power plants. The cohort started with 114,900 participants and increased to 204,103 participants. Since 2003, informed consent has been obtained by mail in the form of opt-out method. By the end of March 2014, 6\% of the participants had not consented, and we stopped following them. The dose information for these workers was provided by the Radiation Dose Registration Center of the REA.

Five study phases were successively conducted from 1990 to 2014 according to the timeline included in Table 1. The final study population at the end of phase V included 204,103 workers. Since 2015, phase VI of the survey has been ongoing.

\subsection{Follow-Up of Vital Status}

\subsubsection{Phases I to $\mathrm{V}$}

The follow-up mortality survey was carried out using residence registration records. Every four years or less, we requested residence registration cards for each worker from the municipal office of their residential address. If the municipal office issued a card or a record of moving to another municipality, we concluded that the subject was still alive and submitted the participant to the next study phase. However, if the municipal office issued a deleted residence card instead of a resident card, we concluded that the subject was deceased and confirmed the cause of the death by record linkage with the National Vital Statistics provided by the Ministry of Health and Welfare (later called the Ministry of Health, Labour and Welfare) of Japan.

As part of the mortality surveys, we conducted lifestyle surveys in 1997 and 2003 in a sub-cohort of approximately 80,000 respondents. The lifestyle questionnaire included items about smoking, alcohol consumption, duration of education, and so on. The results showed that smoking was a confounding factor in the relationship between radiation dose and cancer mortality.

\subsubsection{Phase VI}

After the completion of the fifth survey, the survey protocol has been modified. The follow-up mortality survey has been conducted using residence registration records with the remaining study cohort. Based on the results obtained from the lifestyle surveys shown above, subjects were extended from the sub-cohort to the entire cohort. To respect the increasing privacy concerns in recent years, an opt-in method has been adopted in 2015 to replace the opt-out method used prior to 2003.

\section{Brief Results}

\subsection{Phase I to V}

The main study cohort of 204,103 workers who had been employed in the nuclear industry was subjected to the mortality survey, including the sub-cohort of 75,442 respondents to the lifestyle survey.

Mortality analysis identified a relationship between the cumulative radiation dose and malignant neoplasms in some cases. However, because the lifestyle analysis identified possible confounding variables, such as smoking, we cannot conclude that low-dose radiation affects the mortality rate in individuals with malignant neoplasms.

\subsection{Phase VI}

Phase VI of the study is ongoing and will be terminated at the end of 2019.

This work was fully funded by Japan's Nuclear Regulation Authority.

*1 1-9-16 Maruishi Dai-ni Building 5F, Kajicho, Chiyoda-ku, Tokyo 101-0044, Japan. 
Table 1 Duration and population for analysis of REA's epidemiological study.

\begin{tabular}{lcc}
\hline \multicolumn{1}{c}{ Study phase } & Duration & Population for analysis \\
\hline Phase I & $1990-1994$ & 114,900 \\
Phase II (first lifestyle survey) & $1995-1999(1997)$ & 119,484 \\
Phase III (second lifestyle survey) & $2000-2004(2003)$ & 200,583 \\
Phase IV & $2005-2009$ & 203,904 \\
Phase V & $2010-2014$ & 204,103 \\
Phase VI & $2015-$ present & Ongoing \\
\hline
\end{tabular}

